\title{
Optimum Tuning of a Gyroscopic Vibration Absorber for Vibration Control of a Vertical Cantilever Beam with Tip Mass
}

\author{
F. Ünker \\ Department of Mechanical Engineering, Gümüşhane University, 29100 Gümüşane, Turkey. \\ O. Çuvalcı \\ Department of Mechanical Engineering, Karadeniz Technical University, 61080 Trabzon, Turkey.
}

\begin{abstract}
(Received 8 October 2015; accepted 26 August 2016)
In this paper, a symmetric gyro with coupling to the tip mass of a beam mounted on a vibrating base is considered. Taking advantage of the angular momentum of the rotating gyroscope, gyrostabilizer systems are expected to become more widely actualized as they provide an effective means of motion control with several significant advantages for various structures. This paper mainly focused on finding optimized stiffness and damping values that minimize the vibration responses with the derivation of the frequency equations of a special combined gyrobeam system. Correctness of the analytical results is verified by numerical simulations. The comparison with the results from the derivation of the corresponding frequency equations shows that the optimized stiffness and damping values are very accurate.
\end{abstract}

\section{INTRODUCTION}

Beams carrying a tip mass with (or without) rotary inertia can find application in the modeling of many engineering fields such as tall buildings, towers, piles, antennas, robotic manipulators, appendages of aircrafts, spacecrafts or vehicles, etc. Hence, there has been a lot of research work on the nonlinear dynamics of such beams subject to various boundary and load conditions over the past few decades due to both theoretic and practical demands. In some previous studies, a Euler-Bernoulli beam model has been used to investigate the problem of a vertical cantilever beam having a concentrated mass and various springs along the beam. ${ }^{1-3}$

Currently, a number of researchers studied and tried to solve the vibration problem due to low stiffness of the lightweight and flexible structures by applying one or more passive dynamic vibration absorbers. The most widely used passive vibration absorbers in engineering are tuned mass absorbers, beam absorbers, pendulum-type absorbers, and liquid absorbers. Cuvalci investigated the dynamic behavior of a beamtip mass-pendulum system subjected to a periodic excitation in the presence of autoparametric interaction between the beam and pendulum. ${ }^{4}$ Ertas et al. investigated experimentally an orientable flexible beam with a tip mass and pendulum. ${ }^{5}$ The passive vibration absorbers can be quite effective and desirable due to their simplicity and low cost; but, since passive vibration absorbers have their limitations, one needs to develop the active vibration absorbers. The passive control method is activated by the structural motion without requiring external force or energy to reduce structural responses and utilizes the motion of the structure at the location of the device. ${ }^{6}$ The active control method requires a considerable amount of external power to operate actuators that supply a control force to the structure. Due to requiring sensors and controller equipments, active control devices are more complex, and they do not have the reliability and robustness compared to passive control techniques. $^{7}$

In recent years, several researchers have conducted studies investigating the complicated motions which appear in a gyrostabilizer, of which there are various types and arrangements. One engineering application of the gyroscopic effect is as an effective means of motion control in many situations, for example stabilization of bicycles, cars, monorails, building wind induced vibrations, and ships. ${ }^{8-12}$ Compared to conventional active mass dampers for wind vibration suppression, gyrostabilizers represent a weight and volume saving. The mechanism does not require any other external source of energy, in which the rotor speed is produced by an electric motor in a rotating gimbal. Therefore, this can be classified as a passive control device in a variation of passive vibration control systems. The gyrostabilizer is effective for bending moments rather than shear forces because the gyrostabilizer utilizes the gyroscopic moment to reduce or eliminate the undesirable motions. This paper considers the motion of a symmetric gyro with coupling to the free end of a beam subjected to a harmonic base excitation, which is supported with a torsional spring and damper. The optimal values of the rotor speed, torsional spring, and damper are theoretically analyzed and derived for a cantilever beam. The relations for the optimal values of the gyrostabilizer are so tuned that this system is more adaptable and has a smaller mass than other conventional control devices with the same ability to be employed for vibration control.

\section{EQUATIONS OF MOTION OF THE GYRO- BEAM SYSTEM FOR SMALL VIBRATION}

Figure 1 shows the beam as a vertical cantilever of length $L$ with an end mass $M_{t}$ to which an additional gyro system was attached at the free end and subjected to a harmonic base excitation at the other end of the beam. The horizontal displacement of base was $z$. The beam was assumed to be initially straight, of length $L$. The horizontal and vertical elastic displacements at the free end were $v$ and $u$, respectively. Due to elastic deformation of the beam, $s$ represented the distance along the arc-length of the beam.

The gyro system consisted of a disk mass $m$, which can 


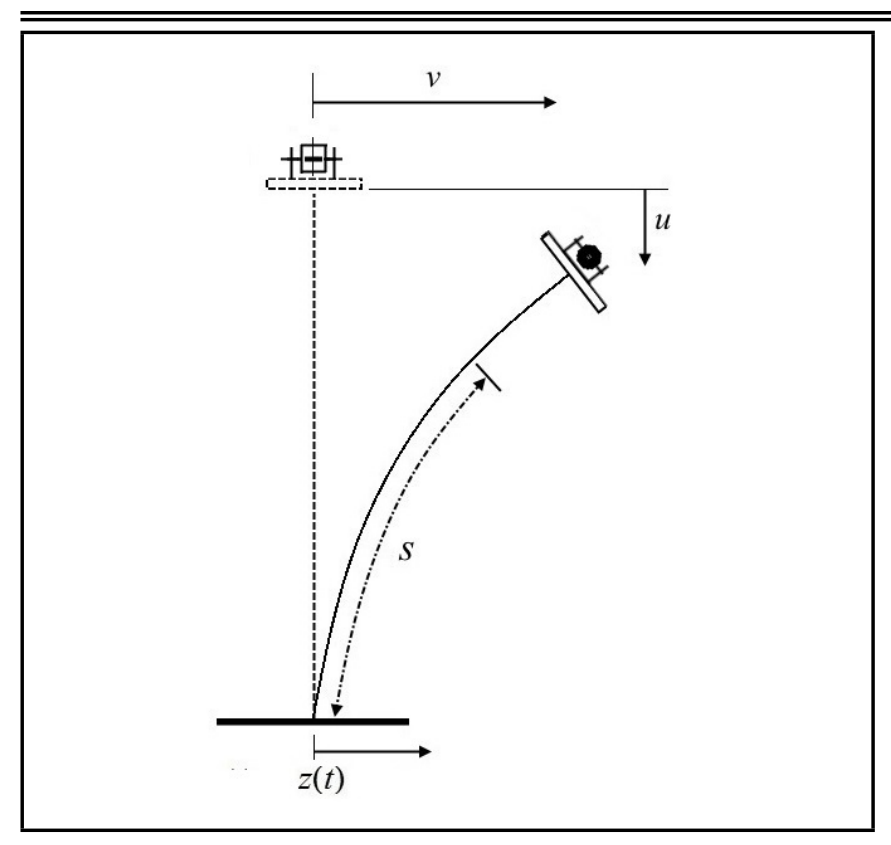

Figure 1. Cantilever model with end mass, gyroscope, and horizontal base excitation.

Table 1. Physical properties of the system.

\begin{tabular}{|l|l|l|}
\hline Symbol & $\begin{array}{l}\text { Numerical } \\
\text { values }\end{array}$ & Description \\
\hline$E$ & $17 \times 10^{9} \mathrm{~N} / \mathrm{m}^{2}$ & Young's modulus \\
\hline$h$ & $200 \mathrm{~mm}$ & beam thickness \\
\hline$b$ & $300 \mathrm{~mm}$ & beam width \\
\hline$L$ & $5 \mathrm{~m}$ & length of the beam \\
\hline$\rho$ & $2300 \mathrm{~kg} / \mathrm{m}^{3}$ & density of the beam \\
\hline$M_{t}$ & $2000 \mathrm{~kg}$ & tip mass \\
\hline$I_{t} / M_{t}$ & $0.2 \mathrm{~m}^{2}$ & $\begin{array}{l}\text { ratio of tip mass moment } \\
\text { of inertia }\end{array}$ \\
\hline$I_{t}$ & $400 \mathrm{~kg} \cdot \mathrm{m}^{2}$ & $\begin{array}{l}\text { tip mass moment of } \\
\text { inertia }\end{array}$ \\
\hline$I$ & $2 \times 10^{-4} \mathrm{~m}^{4}$ & $\begin{array}{l}\text { geometrical moment of } \\
\text { inertia }\end{array}$ \\
\hline$g$ & $9.81 \mathrm{~m} / \mathrm{s}^{2}$ & gravitational acceleration \\
\hline$A=b \cdot h$ & $0.06 \mathrm{~m}{ }^{2}$ & area of cross section \\
\hline$c$ & $80-160 \mathrm{~N} \cdot \mathrm{m} \cdot \mathrm{s} / \mathrm{rad}$ & damping coefficient \\
\hline$k$ & $0.25 I_{o} \omega_{n}^{2}-4 I_{o} \omega_{n}^{2}$ & $\begin{array}{l}\text { stiffness of torsion } \\
\text { spring }\end{array}$ \\
\hline$m$ & $100 \mathrm{~kg}$ & disk mass of gyroscope \\
\hline$r$ & $0.25 \mathrm{~m}$ & radius of disk \\
\hline$I_{p}=m \cdot r^{2} / 2$ & $3.125 \mathrm{~kg} \cdot \mathrm{m}^{2}$ & rotary inertia of disk \\
\hline$I_{o}=I_{p} / 2$ & $1.5625 \mathrm{~kg} \cdot \mathrm{m}^{2}$ & $\begin{array}{l}\text { mass moment of inertia } \\
\text { of disk }\end{array}$ \\
\hline$\Omega$ & $0-20000 \mathrm{rpm}$ & rotating speed of disk \\
\hline & & \\
\hline
\end{tabular}

spin freely about its geometric axis (gimbal) mounted to the tip mass of the beam as shown in Fig. 2. The disk mass, $m$ of gyro at the free end was assumed to have angular speed $\Omega$, and the rotation of the gimbal of this disk was also resisted by torsional spring and damping torque defined by $k \theta$ and $c \dot{\theta}$, respectively.

Equations of the beam and gyro have been expressed in Ünker and Çuvalcı (see Eqs. (16) and (17)) as Eq. (1) and Eq (2). ${ }^{13}$

After the rearrangement, Eq. (1) became Eq. (3) in which:

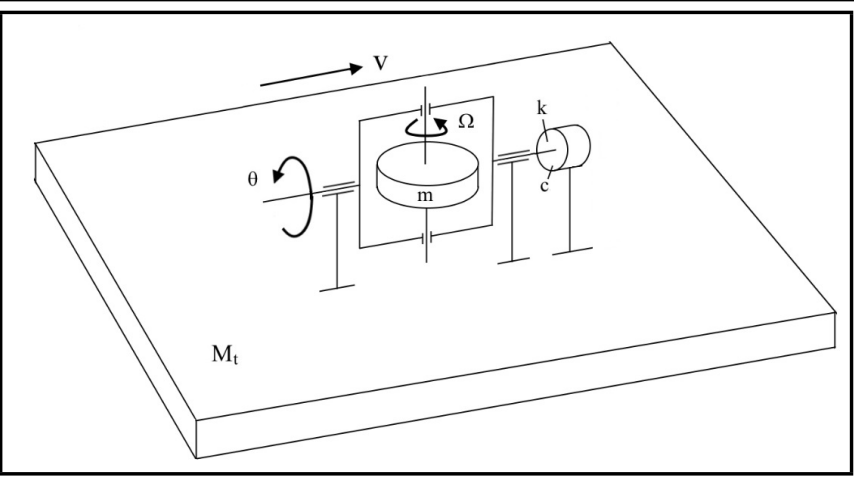

Figure 2. Gyro model with end mass $M_{t}$, massless torsional spring and damper.

$$
\begin{aligned}
\alpha_{1} & =\rho A G_{1}+m+M_{t}+I_{t} G_{5}{ }^{2} ; \\
\alpha_{2} & =\rho A G_{3}+\left(m+M_{t}\right) G_{4}{ }^{2}+I_{t} G_{5}{ }^{4} ; \\
\alpha_{3} & =\frac{1}{4} I_{t} G_{5}{ }^{6} ; \\
\alpha_{4} & =E I G_{6}-\rho A g G_{9}-\left(m+M_{t}\right) g G_{4} ; \\
\alpha_{5} & =2 E I G_{7} ; \\
\alpha_{6} & =\frac{3}{4} E I G_{8} ; \\
\gamma & =\rho A G_{2}+m+M_{t} ;
\end{aligned}
$$

where the constants from $G_{1}$ to $G_{9}$ are given in Table 2 .

Neglecting the terms of higher power for small vibrations $(v \approx 0)$, Eqs. (2) and (3) were reduced to:

$$
I_{0} \ddot{\theta}-\frac{1}{2}\left(I_{p}-I_{o}\right)\left(\dot{v} G_{5}\right)^{2} \sin 2 \theta-I_{p} \Omega \dot{v} G_{5} \cos \theta+c \dot{\theta}+k \theta=0 ;
$$

$$
\begin{aligned}
{\left[\alpha_{1}\right.} & \left.+G_{5}^{2}\left(I_{o} \cos ^{2} \theta+I_{p} \sin ^{2} \theta\right)\right] \ddot{v} \\
& +\left[\alpha_{2}+G_{5}{ }^{4}\left(I_{o} \cos ^{2} \theta+I_{p} \sin ^{2} \theta\right)\right] v \dot{v}^{2}+\alpha_{4} v \\
& +G_{5}\left[G_{5}\left(I_{p}-I_{0}\right) \dot{v} \theta \sin 2 \theta+I_{p} \Omega \theta \cos \theta\right]=-\gamma \ddot{z}
\end{aligned}
$$

\section{OPTIMAL TUNING ABOUT THE EQUILIBRIUM POSITION}

The easiest way to approach this problem was from the point of view of energy. We chose the kinetic to be zero when the tip mass was at the bottom of its swing. Setting $\dot{v} \approx 0$ and $\dot{\theta} \approx 0$ about the equilibrium position, Eqs. (5) and (6) reduced to:

$$
I_{o} \ddot{\theta}-I_{p} \Omega \dot{v} G_{5} \cos \theta+c \dot{\theta}+k \theta=0
$$

$$
\begin{aligned}
{\left[\alpha_{1}+G_{5}^{2}\left(I_{o} \cos ^{2} \theta\right.\right.} & \left.\left.+I_{p} \sin ^{2} \theta\right)\right] \ddot{v} \\
& +\alpha_{4} v+G_{5} I_{p} \Omega \theta \cos \dot{\theta}=-\gamma \ddot{z}
\end{aligned}
$$

The natural frequencies of gyro and beam were determined as follows, respectively:

$$
\omega_{g}^{2}=\frac{k}{I_{o}} ;
$$

$$
\omega_{n}^{2}=\frac{\alpha_{4}}{\alpha_{1}+G_{5}^{2}\left(I_{o} \cos ^{2} \theta+I_{p} \sin ^{2} \theta\right)} .
$$




$$
\begin{aligned}
& \left\{\begin{array}{c}
\rho A G_{1}+m+M_{t}+I_{t} G_{5}{ }^{2}+ \\
{\left[\rho A G_{3}+\left(m+M_{t}\right) G_{4}{ }^{2}+I_{t} G_{5}{ }^{4}\right] v^{2}} \\
+\frac{1}{4} I_{t} G_{5}{ }^{6} v^{4} \\
+\left(G_{5}+\frac{1}{2} v^{2} G_{5}{ }^{3}\right)^{2}\left(I_{o} \cos ^{2} \theta+I_{p} \sin ^{2} \theta\right)
\end{array}\right\} \ddot{v}+\left[\rho A G_{3}+\left(m+M_{t}\right) G_{4}{ }^{2}+I_{t} G_{5}{ }^{4}+\frac{1}{2} I_{t} G_{5}{ }^{6} v^{2}\right] v \dot{v}^{2} \\
& +\left[E I G_{6}-\rho A g G_{9}-\left(m+M_{t}\right) g G_{4}+2 E I G_{7} v^{2}+\frac{3}{4} E I G_{8} v^{4}\right] v \\
& +\left(G_{5}+\frac{1}{2} v^{2} G_{5}{ }^{3}\right)\left[\begin{array}{c}
\left(I_{p}-I_{0}\right)\left(\dot{v} G_{5}+\frac{1}{2} v^{2} \dot{v} G_{5}^{3}\right) \dot{\theta} \sin 2 \theta \\
+I_{o} v \dot{v}^{2} G_{5}{ }^{3} \cos ^{2} \theta \\
+I_{p} v \dot{v}^{2} G_{5}{ }^{3} \sin ^{2} \theta \\
+I_{p} \Omega \dot{\theta} \cos \theta
\end{array}\right]=-\left(\rho A G_{2}+m+M_{t}\right) \ddot{z} ; \\
& I_{0} \ddot{\theta}-\frac{1}{2}\left(I_{p}-I_{o}\right)\left(\dot{v} G_{5}+\frac{1}{2} v^{2} \dot{v} G_{5}{ }^{3}\right)^{2} \sin 2 \theta-I_{p} \Omega \quad\left(\dot{v} G_{5}+\frac{1}{2} v^{2} \dot{v} G_{5}{ }^{3}\right) \cos \theta+c \dot{\theta}+k \theta \quad=\quad 0 \\
& {\left[\alpha_{1}+\alpha_{2} v^{2}+\alpha_{3} v^{4}+\left(G_{5}+\frac{1}{2} v^{2} G_{5}^{3}\right)^{2}\left(I_{o} \cos ^{2} \theta+I_{p} \sin ^{2} \theta\right)\right] \ddot{v}} \\
& +\left[\alpha_{2}+2 \alpha_{3} v^{2}+G_{5}{ }^{3}\left(G_{5}+\frac{1}{2} v^{2} G_{5}{ }^{3}\right)\left(I_{o} \cos ^{2} \theta+I_{p} \sin ^{2} \theta\right)\right] v \dot{v}^{2}+\left(\alpha_{4}+\alpha_{5} v^{2}+\alpha_{6} v^{4}\right) v \\
& +\left(G_{5}+\frac{1}{2} v^{2} G_{5}{ }^{3}\right)\left[\left(I_{p}-I_{o}\right) \dot{v}\left(G_{5}+\frac{1}{2} v^{2} G_{5}{ }^{3}\right) \dot{\theta} \sin 2 \theta+I_{p} \Omega \dot{\theta} \cos \theta\right]=-\gamma \ddot{z} .
\end{aligned}
$$

Table 2. Constants of the equation of motion of the gyro-column system in SI units.

\begin{tabular}{|c|c|c|c|c|c|c|c|c||}
\hline $\begin{array}{c}G_{1} \\
=L\left(\frac{3}{2}-\frac{4}{\pi}\right)\end{array}$ & $\begin{array}{c}G_{2} \\
=\frac{L}{\pi}(\pi-2)\end{array}$ & $\begin{array}{c}G_{3} \\
=\frac{\pi^{2}}{384 L}\left(2 \pi^{2}-9\right)\end{array}$ & $\begin{array}{c}G_{4} \\
=\frac{\pi^{2}}{8 L}\end{array}$ & $\begin{array}{c}G_{5} \\
=\frac{\pi}{2 L}\end{array}$ & $\begin{array}{c}G_{6} \\
=\frac{\pi^{4}}{32 L^{3}}\end{array}$ & $\begin{array}{c}G_{7} \\
=\frac{\pi^{6}}{512 L^{5}}\end{array}$ & $\begin{array}{c}G_{8} \\
=\frac{\pi^{8}}{4096 L^{7}}\end{array}$ & $\begin{array}{c}G_{9} \\
=\frac{1}{16}\left(\pi^{2}-4\right)\end{array}$ \\
\hline 1.1338 & 1.8169 & 0.0552 & 0.2467 & 0.3142 & 0.0244 & $6.0087 \mathrm{e}-4$ & $2.9652 \mathrm{e}-5$ & 0.3669 \\
\hline
\end{tabular}

Assuming a harmonic disturbing base excitation $z=\bar{z} e^{j \omega t}$, the responses were written as angular frequency $\omega$ :

$$
\theta=\bar{\theta} e^{j \omega t} \quad \text { and } \quad \mathrm{v}=\overline{\mathrm{v}} e^{j \omega t} .
$$

Therefore, Eqs. (7) and (8) were rewritten in the matrix form:

$$
\begin{gathered}
\bar{\theta}\left[\begin{array}{c}
j \omega G_{5} I_{p} \Omega \cos \theta \\
-I_{o} \omega^{2}+j c \omega+k
\end{array}\right]+ \\
\overline{\mathrm{v}}\left[\begin{array}{c}
-\omega^{2}\left[\alpha_{1}+G_{5}{ }^{2}\left(I_{o} \cos ^{2} \theta+I_{p} \sin ^{2} \theta\right)\right]+\alpha_{4} \\
-j \omega I_{p} \Omega G_{5} \cos \theta
\end{array}\right]= \\
{\left[\begin{array}{c}
\omega^{2} \gamma \bar{z} \\
0
\end{array}\right]}
\end{gathered}
$$

Solving these equations yielded Eqs. (13) and (14). The complex Eqs. (13) and (14) were transformed to the form of Eqs. (15) and (16)

\subsection{The Undamped Gyroscopic Vibration Absorber}

In order to reach the minimum tip mass displacement $|\overline{\mathrm{v}}|$, the natural frequency of the added gyro-spring system itself should have been the same as the excitation frequency $\left(\omega^{2}=\right.$ $\frac{k}{I_{0}}=\omega_{g}^{2}$ ), and by eliminating term of damping, Eq. (16) was then:

$$
|\bar{\theta}|=\gamma \bar{z} \omega^{3} \sqrt{\frac{\left(I_{p} \Omega G_{5} \cos \theta\right)^{2}}{\left[\omega^{2}\left(I_{p} \Omega G_{5} \cos \theta\right)^{2}\right]^{2}}}
$$

Autoparametric resonance occured when $\left(\omega^{2}=\omega_{n}^{2}=\omega_{g}^{2}\right)$. Upon assuming $\theta=\bar{\theta} e^{j \omega t}=\bar{\theta}_{\max }$ for the equation of a stable motion at $\omega \leq \omega_{n}$, Eq. (17) corresponded to:

$$
\left|\bar{\theta}_{\max }\right|=\frac{\gamma \bar{z}_{n} \omega}{I_{p} \Omega G_{5} \cos \bar{\theta}_{\max }} .
$$

The minimum disk speed $\Omega_{\min }$ was chosen carefully to eliminate the instability at $\omega \leq \omega_{n}$. Since a minimum disk speed $\Omega_{\min }$ is required for a stable motion, Eq. (18) was modified into the following form:

$$
\bar{\theta}_{\max } \cos \bar{\theta}_{\max }= \pm \frac{\omega_{n} \gamma \bar{z}}{I_{p} G_{5} \Omega_{\min }} .
$$

Supposing that a minimum disk speed was required for a stable motion, this transformed to a minimum disk speed equation:

$$
\Omega_{\min }= \pm \frac{\omega_{n} \gamma \bar{z}}{I_{p} G_{5} \bar{\theta}_{\max } \cos \bar{\theta}_{\max }}
$$

Figure 3 shows that the theoretical limit of the amplitude at resonance is one when absolute value of the rotation $\bar{\theta}_{\max }$ approaches $0.86 \mathrm{rad}$. Supposing that $\left|\bar{\theta}_{\max } \cos \bar{\theta}_{\text {max }}\right|=0.5611$ and $\left|\bar{\theta}_{\max }\right|=0.86 \mathrm{rad}$ for $-\pi / 2 \leq \bar{\theta} \leq \pi / 2$, Eq. (20) was rewritten as:

$$
\Omega_{\text {min }}=1.7822 \frac{\omega_{n} \gamma \bar{z}}{I_{p} G_{5}}
$$

where Eq. (10) was then:

$$
\omega_{n}=\sqrt{\frac{\alpha_{4}}{\alpha_{1}+G_{5}^{2}\left(0.426 I_{o}+0.574 I_{p}\right)}} .
$$




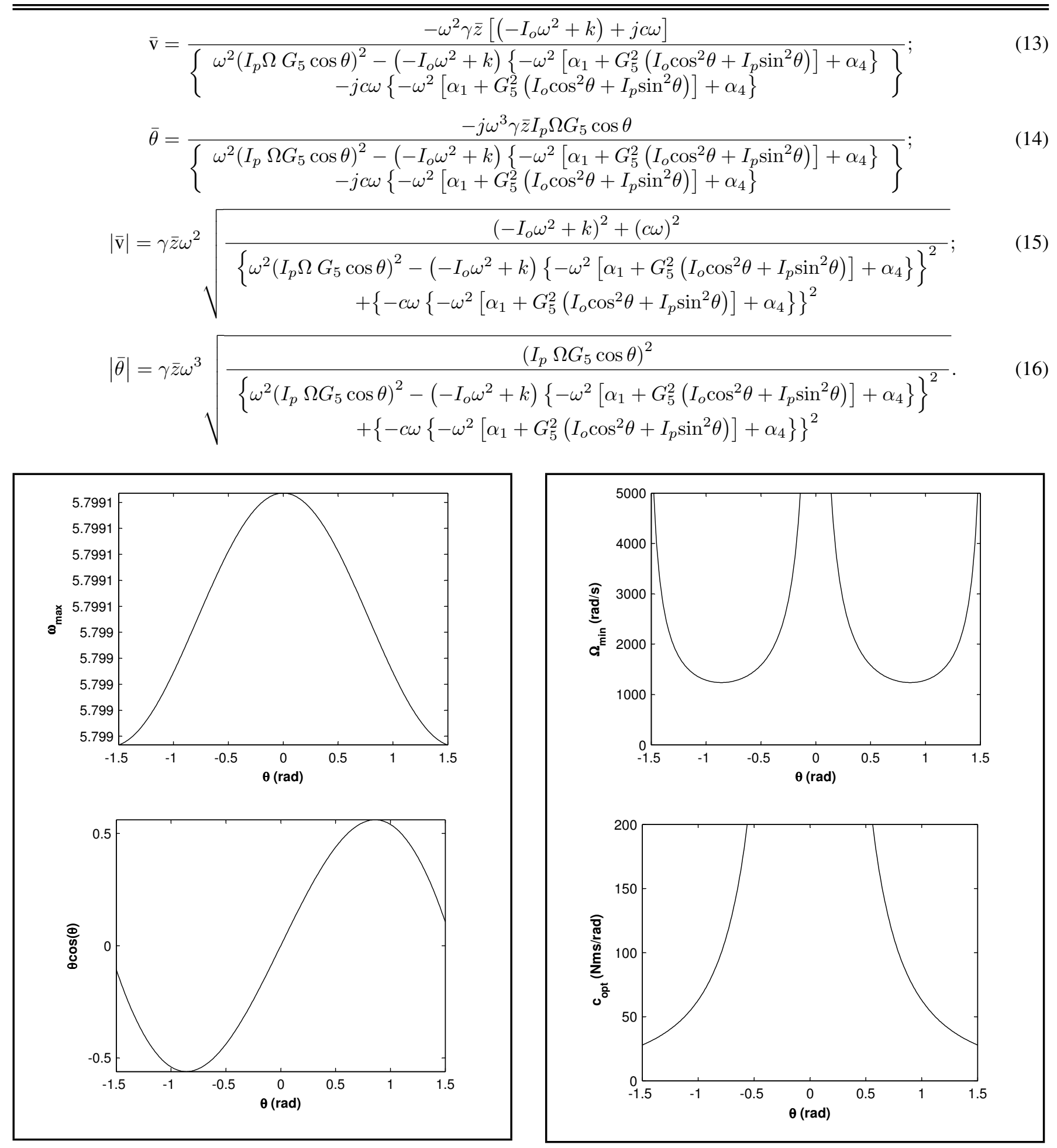

Figure 3. The theoretical limit curves: natural frequency rad/s (top), gyro response rad (bottom).

\subsection{The Damped Gyroscopic Vibration Absorber}

With the help of Eq. (16), it was rewritten as Eq (23).

After setting $\omega^{2}=\omega_{n}^{2}=\omega_{g}^{2}$ and $\Omega=\Omega_{\min }$ in order to reach the optimum tuning, Eq. (23) was expressed as Eq. (24) Since Eq. (24) was an undefined form, with the help of Eq. (14) and using L'Hospital's rule, optimum damping was obtained Eq. (25).

As seen from Fig. 4, minimum disk speed was generated at the angle of $0.86 \mathrm{rad}$, which achieved a stable motion of its swing. Throughout the next entire analysis, the angle

Figure 4. The theoretical limit curves: minimum disk speed $\Omega_{\min } \mathrm{rad} / \mathrm{s}$ (top), Optimal damper tuning Nms/rad (bottom).

of 0.86 rad was preferred as an allowable maximum precession. Hence, setting $\bar{\theta}_{\text {opt }}=\left|\bar{\theta}_{\text {max }}\right|=0.86 \mathrm{rad}$ and $\Omega_{\text {min }}=$ $1.7822 \frac{\omega_{n} \gamma \bar{z}}{I_{p} G_{5}}$ to eliminate the instability due to the natural frequency of the added gyroscope resulted in optimal damping coefficient. Figure 5 shows the possible maxima and minima of the theoretical frequency responses of a beam and gyroscope obtained from Eqs. (15) and (16), respectively. In the figure, it was fairly proven that optimal damping and spring coefficients do not allow the precession grow over 0.86 rad for a stable motion. 


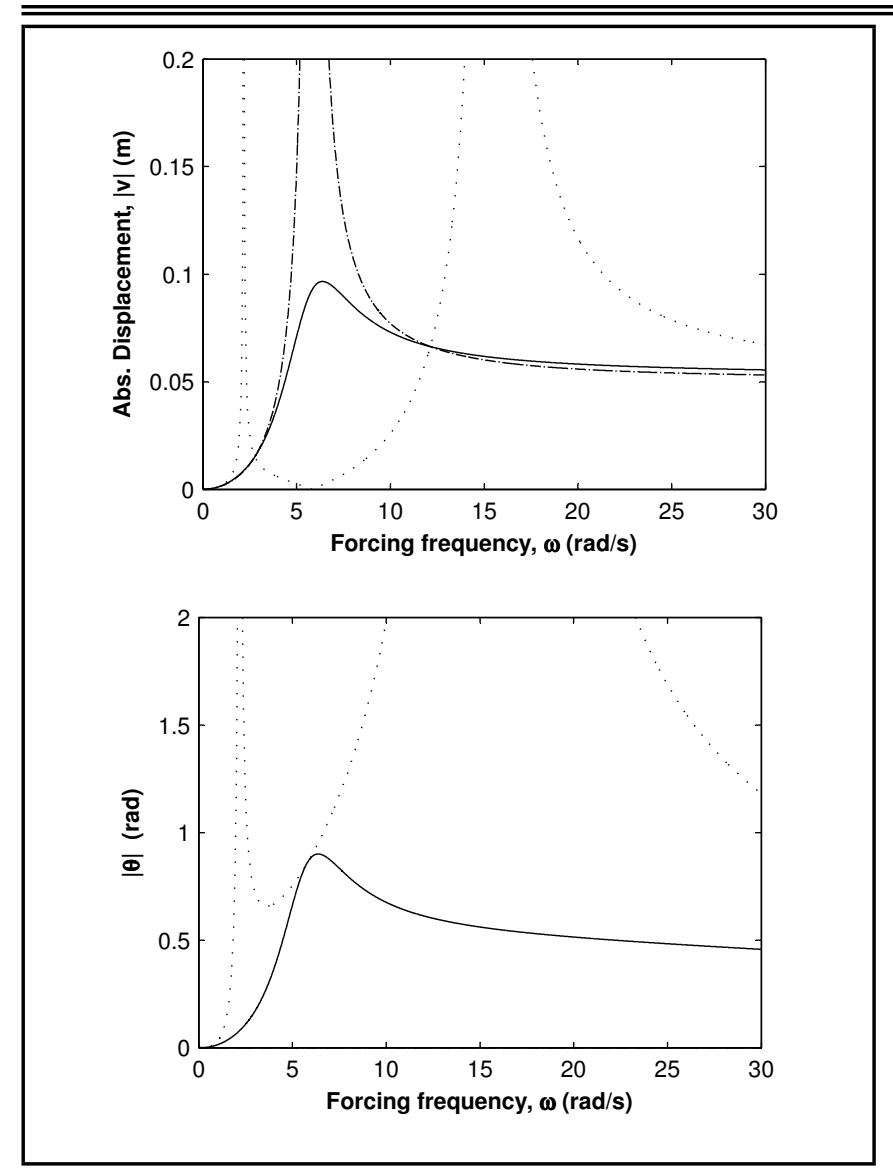

Figure 5. The analitical frequency response curves of tip of beam with $\bar{z}=$ $0.05 \mathrm{~m}$; for (dotted) $\Omega=\Omega_{m i n}, k=I_{0} \omega_{n}^{2}$ and $c=0$, for (solid) $\Omega=$ $\Omega_{\text {min }}, k=I_{0} \omega_{n}^{2}$ and $c=c_{o p t}$, and for (dash-dot) $\Omega=0$.

\section{NUMERICAL SIMULATIONS}

In the following calculations, a rectangle-cross beam was considered with thickness $h=200 \mathrm{~mm}$, width $b=300 \mathrm{~mm}$, length $L=5 \mathrm{~m}$, density $\rho=2300 \mathrm{~kg} / \mathrm{m}^{3}$, and Young's modulus along the axial direction $E=17 \times 109 \mathrm{~N} / \mathrm{m}^{2}$. Equations (1) and (2) were solved by using a MATLAB software tool that involves the fourth-order Runge-Kutta method. The parameters of the numerical example are given in Table 1. In order to identify the dynamical behavior, the time history was simulated with the time step size of $0.05 \mathrm{~s}$ and zero initial conditions.

\subsection{The Undamped Gyroscopic Vibration Absorber}

Figure 6 shows the rotational speed curves for cases: (blue) $0.25 \omega_{n}^{2}=\omega_{g}^{2}$, (green) $\omega_{n}^{2}=\omega_{g}^{2}$, and (red) $\omega_{n}^{2}=0.25 \omega_{g}^{2}$, respectively. The results presented in this figure all indicated that the gyro system had significantly reduced the vibration of a beam at the free end by increasing the rotating speed $\Omega$ of a disk of a gyro. The tip response of the beam for case (red) $\omega_{n}^{2}=0.25 \omega_{g}^{2}$ was effectively reduced over minimum rotational speed $\left(\Omega_{\min }\right)$. However, optimal performance was achieved for the case of (green) $\omega_{n}^{2}=\omega_{g}^{2}$, which generated less displacement than the response of (red) $\omega_{n}^{2}=0.25 \omega_{g}^{2}$ as seen from Fig. 7. Consequently, the case of (green) $\omega_{n}^{2}=\omega_{g}^{2}$ was preferred as an optimum frequency rate, which agrees with the analytical solutions.

As the disk speed was increased, the location of the maximum frequency of the stable motion changed, as seen from Fig. 8. By the damping effect of the angular momentum of

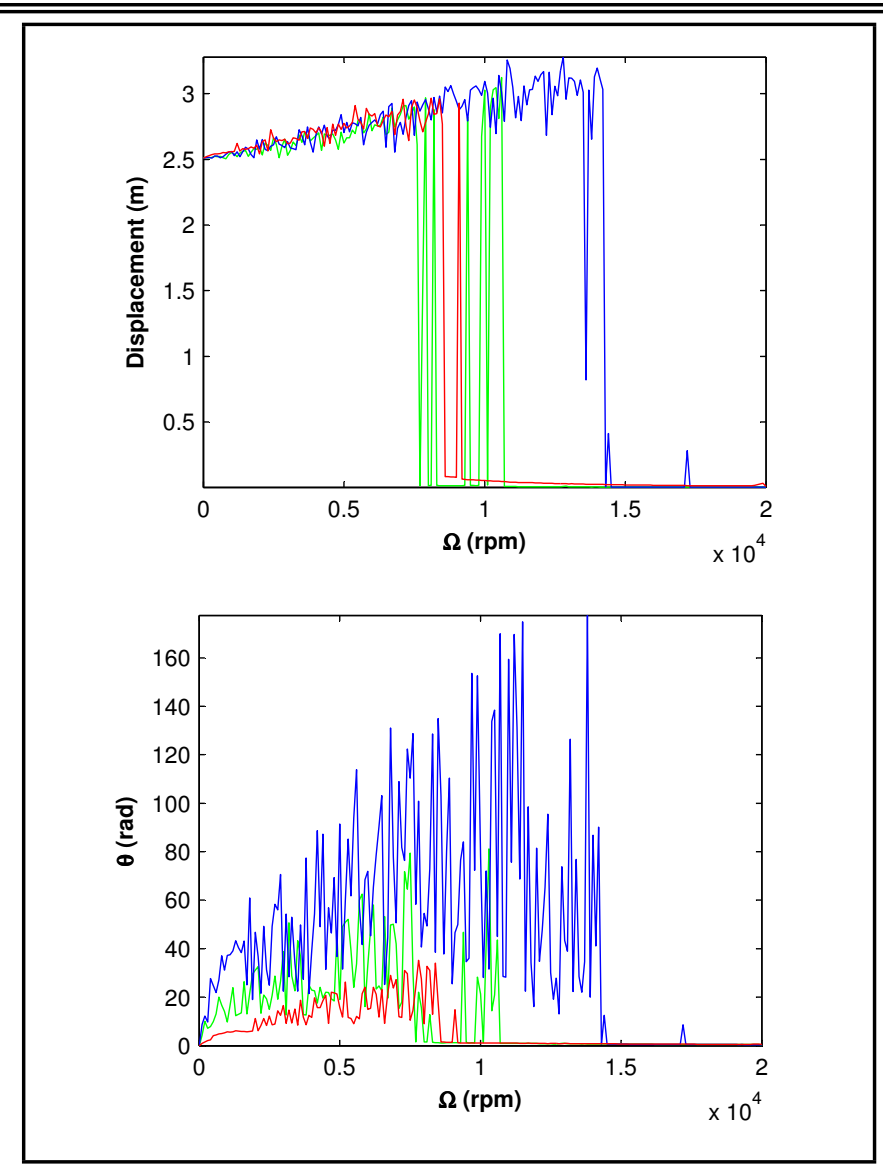

Figure 6. Rotational speed response curves: tip response of beam (top), gyro response (bottom) for (blue) $0.25 \omega_{n}^{2}=\omega_{g}^{2}$, (green) $\omega_{n}^{2}=\omega_{g}^{2}$, and (red) $\omega_{n}^{2}=0.25 \omega_{g}^{2}$ with the base excitation of $z=0.05 \cos \left(\omega_{n} t\right)$.

the gyro, the reachable frequency of a stable motion increased compared to the case for the minimum rotational speed in Fig. 7. However, the peak with low frequency was found to increase the amplitude of displacement as the disk speed was increased.

\subsection{The Damped Gyroscopic Vibration Absorber}

In the case of the base excitation with $z=0.05 \cos (\omega t)$, Figs. 9 and 10 show frequency responses of the beam (top) and the gyro (bottom) at the free end for the damping coefficients of $(\nabla) c=80 \mathrm{~N} \cdot \mathrm{m} \cdot \mathrm{s} / \mathrm{rad},(\square) c=120 \mathrm{~N} \cdot \mathrm{m} \cdot \mathrm{s} / \mathrm{rad}$, and (o) $c=$ $160 \mathrm{~N} \cdot \mathrm{m} \cdot \mathrm{s} / \mathrm{rad}$, respectively. For different rotational speeds, it was seen that the case of $c=80 \mathrm{~N} \cdot \mathrm{m} \cdot \mathrm{s} / \mathrm{rad}$ was approximately optimum damping coefficient obtained from Fig. 4 corresponding to $\bar{\theta}_{\text {opt }}(0.86 \mathrm{rad})$. When damping coefficient was increased, the frequency response of the beam increased compared to the optimum damping $c=80 \mathrm{~N} \cdot \mathrm{m} \cdot \mathrm{s} / \mathrm{rad}$; however, the frequency response of the gyro for $c=80 \mathrm{~N} \cdot \mathrm{m} \cdot \mathrm{s} / \mathrm{rad}$ had high values and the frequency response of the beam jumped into an unstable zone at low frequencies when the rotational speed was increased, as seen in Fig. 10. It may be fairly concluded and proven that optimizing the coefficients of damper and spring would be able to suppress the undesired motions of beam and gyro.

\section{CONCLUSIONS}

This paper provides some analytical equations to investigate the optimum damping and spring coefficient of a gyro-beam system. Optimum spring and damper parameters for minimum 


$$
\begin{aligned}
& c^{2}=\frac{\left(\gamma \bar{z} \omega^{3}\right)^{2} \frac{\left(I_{p} \Omega G_{5} \cos \theta\right)^{2}}{(\bar{\theta})^{2}}-\left\{\begin{array}{c}
\omega^{2}\left(I_{p} \Omega G_{5} \cos \theta\right)^{2}-\left(-I_{o} \omega^{2}+k\right) \\
\cdot\left\{-\omega^{2}\left[\alpha_{1}+G_{5}{ }^{2}\left(I_{o} \cos ^{2} \theta+I_{p} \sin ^{2} \theta\right)\right]+\alpha_{4}\right\}
\end{array}\right\}^{2}}{\left\{-\omega\left\{-\omega^{2}\left[\alpha_{1}+G_{5}{ }^{2}\left(I_{o} \cos ^{2} \theta+I_{p} \sin ^{2} \theta\right)\right]+\alpha_{4}\right\}\right\}^{2}} ; \\
& c^{2}=\frac{\left(\gamma \bar{z} \omega_{n}^{3}\right)^{2} \frac{\left(I_{p} \Omega_{\min } G_{5} \cos \theta\right)^{2}}{(\bar{\theta})^{2}}-\left[\omega_{n}^{2}\left(I_{p} \Omega_{\min } G_{5} \cos \theta\right)^{2}\right]^{2}}{\left\{-\omega_{n}\left\{-\omega_{n}^{2}\left[\alpha_{1}+G_{5}^{2}\left(I_{o} \cos ^{2} \theta+I_{p} \sin ^{2} \theta\right)\right]+\alpha_{4}\right\}\right\}^{2}}=\frac{0}{0} ; \\
& c_{o p t}=\frac{\sqrt{9\left(\gamma \bar{z} \omega_{n}\right)^{2} \frac{\left(I_{p} \Omega_{\text {min }} G_{5} \cos \bar{\theta}_{o p t}\right)^{2}}{\left(\bar{\theta}_{o p t}\right)^{2}}+4\left(I_{p} \Omega_{m i n} G_{5} \cos \bar{\theta}_{o p t}\right)^{4}}}{2 \omega_{n}\left[\alpha_{1}+G_{5}^{2}\left(I_{o} \cos ^{2} \bar{\theta}_{o p t}+I_{p} \sin ^{2} \bar{\theta}_{o p t}\right)\right]} .
\end{aligned}
$$
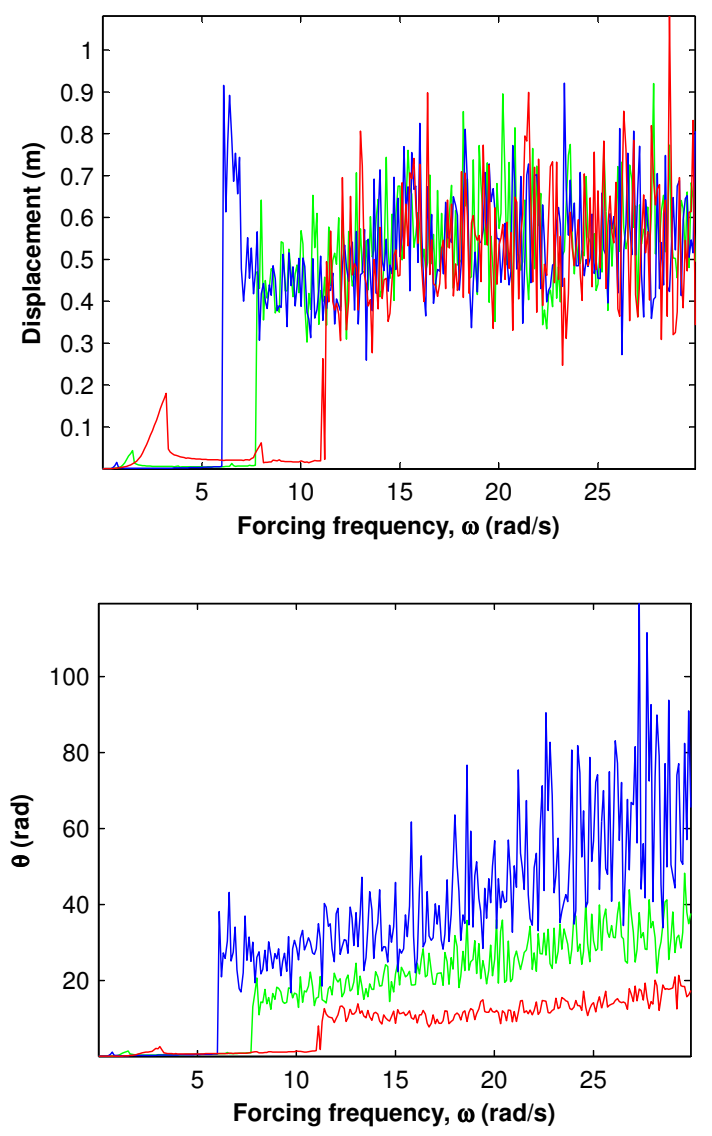

Figure 7. Frequency response curves for $\Omega=15000 \mathrm{rpm}$ : tip response of beam (top), gyro response (bottom) for (blue) $0.25 \omega_{n}^{2}=\omega_{g}^{2}$, (green) $\omega_{n}^{2}=$ $\omega_{g}^{2}$, and (red) $\omega_{n}^{2}=0.25 \omega_{g}^{2}$ with the base excitation of $z=0.05 \cos \left(\omega_{n} t\right)$.

response are obtained from frequency equations of the system. The displacement response of case (green) $\omega_{n}=\omega_{g}$ is effectively reduced up to the resonant frequency. The gyroscope can jump into unstable zone when the stiffness is not taken properly. Hence, the optimum spring coefficient $\left(k=I_{0} \omega^{2}\right)$ is chosen to reach the optimum relations for the minimum rotational speed and an optimum damping. Then, setting $\omega=\omega_{n}=\omega_{g}$ and $\Omega=\Omega_{\min }$ to eliminate the instability due to the natural frequency of the added gyro-spring system results in optimal damping at the resonance frequency.

The study clearly indicates that the tip response of a beam is influenced with the disk speed of the gyro but also depends fairly on the optimum damping coefficient. The numerical results agree with the analytical solution of the damping coefficient corresponding to the minimum disk speed. Therefore, the cooperation of the optimum damping coefficient with the
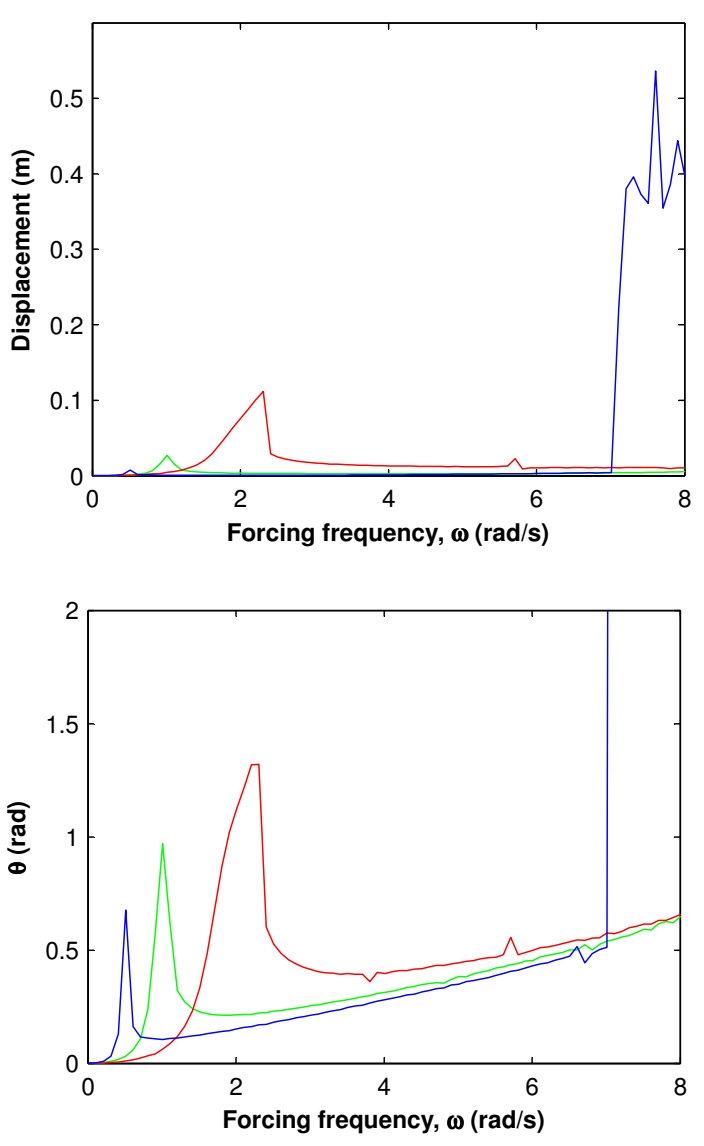

Figure 8. Frequency response curves for $\Omega=20000 \mathrm{rpm}$ : tip response of beam (top), gyro response (bottom) for (blue) $0.25 \omega_{n}^{2}=\omega_{g}^{2}$, (green) $\omega_{n}^{2}=$ $\omega_{g}^{2}$, and (red) $\omega_{n}^{2}=0.25 \omega_{g}^{2}$ with the base excitation of $z=0.05 \cos \left(\omega_{n} t\right)$.

disk speed of the gyro is not negligible, and the minimum disk speed at the resonance frequency should always be considered in such calculations.

\section{CONFLICT OF INTERESTS}

The authors declare that there is no conflict of interests regarding the publication of this paper.

\section{ACKNOWLEDGEMENTS}

This study was mainly supported by the Scientific and Technological Research Council of Turkey (TUBITAK) under Grant no: 114M760. 


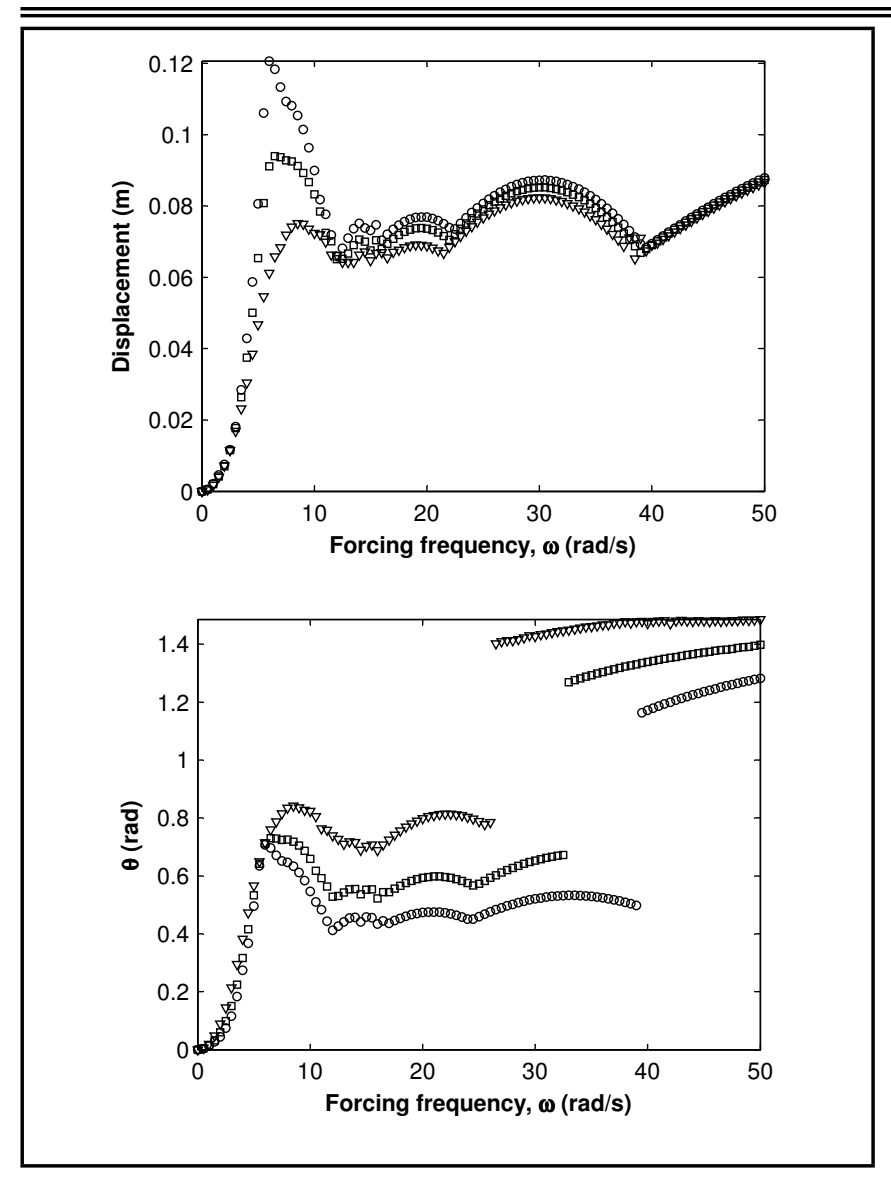

Figure 9. Frequency response curves for $\omega_{n}^{2}=\omega_{g}^{2}$ and $\Omega=10000 \mathrm{rpm}$ : tip response of beam (top), gyro response (bottom) for $(\nabla) c=80 \mathrm{~N} \cdot \mathrm{m} \cdot \mathrm{s} / \mathrm{rad}$, ( $\square$ ) $c=120 \mathrm{~N} \cdot \mathrm{m} \cdot \mathrm{s} / \mathrm{rad}$, and (o) $c=160 \mathrm{~N} \cdot \mathrm{m} \cdot \mathrm{s} / \mathrm{rad}$ with the base excitation of $z=0.05 \cos \left(\omega_{n} t\right)$

\section{REFERENCES}

1 Kim, C. S., and Dickinson, S. M. On the analysis of laterally vibrating slender beams subject to various complicating effects, Journal of Sound and Vibration, 122 (3), 441-455, (1988). https://dx.doi.org/10.1016/S0022-460X(88)800932

2 Liu, W. H., and Huang, C.-C. Free vibration of restrained beam carrying concentrated masses, Journal of Sound and Vibration, 123 (1), 31-42, (1988). https://dx.doi.org/10.1016/S0022-460X(88)80075-0

3 To, C. W. S. Vibration of a cantilever beam with a base excitation and tip mass, Journal of Sound and Vibration, 83 (4), 445-460, (1982). https://dx.doi.org/10.1016/S0022$460 X(82) 80100-4$

4 Cuvalci, O. The effect of detuning parameters on the absorption region for a coupled system: a numerical and experimental study. Journal of Sound and Vibration, 229 (4), 837-857, (2000). https://dx.doi.org/10.1006/jsvi.1999.2526

5 Ertas, A., Cuvalci, O., and Ekwaro-Osire S. Performance of pendulum absorber for a nonlinear system of varying orientation, Journal of Sound and Vibration, 229 (4), 913-933, (2000). https://dx.doi.org/10.1006/jsvi.1999.2521

6 Soong, T. T., and Dargush, G. F. Passive Energy Dissipation System in Structural Engineering, Wiley, New York, NY, USA, (1997).
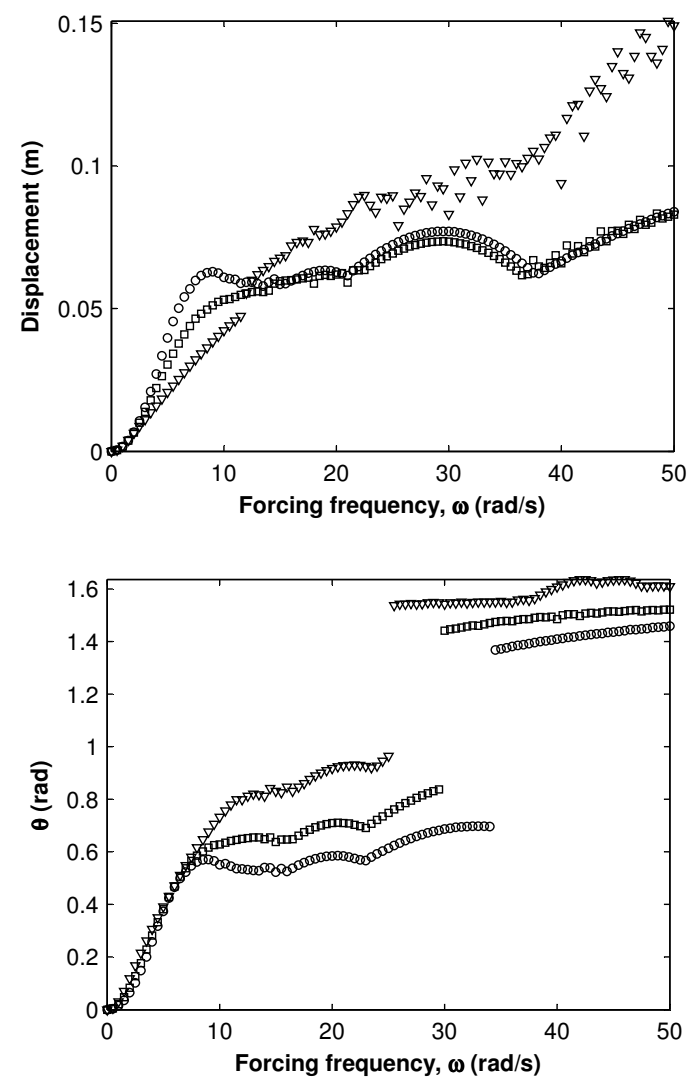

Figure 10. Frequency response curves for $\omega_{n}^{2}=\omega_{g}^{2}$ and $\Omega=15000 \mathrm{rpm}$ : tip response of beam (top), gyro response (bottom) for $(\nabla) c=80 \mathrm{~N} \cdot \mathrm{m} \cdot \mathrm{s} / \mathrm{rad}$, ( $\square$ ) $c=120 \mathrm{~N} \cdot \mathrm{m} \cdot \mathrm{s} / \mathrm{rad}$, and (o) $c=160 \mathrm{~N} \cdot \mathrm{m} \cdot \mathrm{s} / \mathrm{rad}$ with the base excitation of $z=0.05 \cos \left(\omega_{n} t\right)$.

7 Soong, T. T., and Spencer, B. F., Jr. Supplemental energy dissipation: state-of-the-art and state-of-the practice, Engineering Structures, 24 (3), 243-259, (2002). https://dx.doi.org/10.1016/S0141-0296(01)00092-X

8 Beznos, A., Formal'sky, A., Gurfinkel, E., Jicharev, D., Lensky, A., Savitsky, K., and Tchesalin, L. Control of Autonomous Motion of Two-Wheel Bicycle With Gyroscopic Stabilization, Proceedings of the IEEE International Conference on Robotics and Automation, Leuven, Belgium, 3, 2670-2675, (1998). https://dx.doi.org/10.1109/ROBOT.1998.680749

9 Schilovski, P. Gyrocar, (1909), Patent No. GB 12,021.

10 Tomlinson, N. Louis Brennan: Inventor Extraordinaire, John Halliwell: Chatham, (1980).

11 Higashiyama, H., Yamada, M., Kazao, Y., and Namiki, M. Characteristics of Active Vibration Control System Using Gyro-Stabilizer, Eng. Struct., 20 (3), 176-183, (1998). https://dx.doi.org/10.1016/S0141-0296(97)00076-X

12 Schlick, E. The Gyroscopic Effect of Flywheels on Board Ship, Transactions of the Institute of Naval Architects, 23, 117-134, (1904).

13 Ünker, F., and Çuvalcı, O. Vibration Control of a Column Using a Gyroscope, Procedia - Social and Behavioral Sciences, 195, 2306-2315, (2015). https://dx.doi.org/10.1016/j.sbspro.2015.06.182T 